\title{
Refractory migraine in a headache clinic population
}

\author{
Pablo Irimia*, Jose-Alberto Palma, Roberto Fernandez-Torron and Eduardo Martinez-Vila
}

\begin{abstract}
Background: Many migraineurs who seek care in headache clinics are refractory to treatment, despite advances in headache therapies. Epidemiology is poorly characterized, because diagnostic criteria for refractory migraine were not available until recently. We aimed to determine the frequency of refractory migraine in patients attended in the Headache Unit in a tertiary care center, according to recently proposed criteria.

Methods: The study population consisted of a consecutive sample of 370 patients (60.8\% females) with a mean age of 43 years (range 14-86) evaluated for the first time in our headache unit over a one-year period (between October 2008 and October 2009). We recorded information on clinical features, previous treatments, Migraine Disability Assessment Score (MIDAS), and final diagnosis.

Results: Overall migraine and tension-type headache were found in $46.4 \%$ and $20.5 \%$ of patients, respectively. Refractory migraine was found in $5.1 \%$ of patients. In refractory migraineurs, the mean MIDAS score was 96 , and $36.8 \%$ were medication-overusers.

Conclusions: Refractory migraine is a relatively common and very disabling condition between the patients attended in a headache unit. The proposed operational criteria may be useful in identifying those patients who require care in headache units, the selection of candidates for combinations of prophylactic drugs or invasive treatments such as neurostimulation, but also to facilitate clinical studies in this patient group.
\end{abstract}

\section{Background}

Migraine is a common and disabling primary headache disorder [1-3]. Despite substantial advances in migraine therapy [4], many patients are considered refractory to treatment. Although the term refractory migraine (RM) has been used in the literature for decades, operational criteria were not defined until recently [5,6].

The Refractory Headache Special Interest Section of the American Headache Society proposed the criteria for both RM and refractory chronic migraine (R-CM) $[6,7]$. According to this definition, refractory migraineurs must meet the International Classification of Headache Disorders, Second Edition (ICHD-2) criteria for migraine or chronic migraine [1]. Headaches need to cause significant interference with function or quality of life despite modification of triggers, lifestyle factors, and adequate trials of acute and preventive medicines. The trials with acute medicines should include both a triptan

\footnotetext{
*Correspondence: pirimia@unav.es

Department of Neurology. Headache Unit. University Clinic of Navarra. Av. Pio XII, 36, Pamplona 31008, Spain
}

and dihydroergotamine (DHE) intranasal or injectable formulation and either nonsteroidal anti-inflammatory drugs (NSAID) or combination analgesics. The definition requires that patients fail adequate trials of preventive medicines with established efficacy, alone or in combination, from more than 2 of 4 drug classes including: beta-blockers, anticonvulsants, tricyclics, and calcium channel blockers. The definition also includes modifiers for the presence or absence of medication overuse headache $(\mathrm{MOH})$, and with or without significant disability, according to the Migraine Disability Assessment Score (MIDAS).

The proportion of patients with RM and R-CM attending headache units seems to be growing [8], but the actual epidemiology of this disorder is unknown [9].

The purpose of this study was to investigate the frequency of refractory migraineurs, according to the proposed criteria for episodic RM and R-CM [6,7] among the patients who seek care for the first time in a headache unit.

\section{Biomed Central}




\section{Methods}

\section{Study Population}

This study was based on a sample of consecutive patients attended for the first time (only new patients) in the headache unit based in the Department of Neurology at the University Clinic of Navarra, during a oneyear period (from October 2008 to October 2009). Patients attended in our headache unit may be referred from primary care, the emergency department, other health professionals, or self-referred. All patients underwent a clinical interview and neurological examination by the first author. Information on clinical features, possible triggers, previous or current preventive and abortive treatments and diagnosis were recorded. Additional investigations to rule out secondary causes of headache (mainly cerebral magnetic resonance imaging) were performed depending on the results of the clinical history and neurological examination. MIDAS was used as the clinical measure of headache-related disability [10]. MIDAS scores can be classified into four severity grades: little or none (0-5), mild (6-10), moderate (11-20), and severe $(\geq 21)$. A standardized questionnaire based on the ICHD-2 was used for new patients attended in the headache unit. The information obtained from the patients was stored in a Headache database for statistical analysis. Each patient or the patient's guardian gave their informed consent and the local Ethics Committee approved the study.

\section{Classification}

Headaches were classified according to the diagnostic criteria of the International Classification of Headache Disorders, second edition (ICHD-2) [1] and revised criteria for chronic migraine and medication overuse headache $(\mathrm{MOH})$ [11]. To define patients with episodic RM and $\mathrm{R}-\mathrm{CM}$, we used the criteria proposed by Schulman et al. [6], which were modified during the validation procedure [7].

\section{Statistics}

Statistical analyses were performed using SPSS version 15.0 for Windows (SPSS Inc, Chicago, IL, USA). For categorical (qualitative) data, the $\chi^{2}$ test was used to check the differences between the two groups. When the expected frequencies were less than 5, Fisher's exact test was performed.

For comparisons of two or more means, the analysis of variance (ANOVA) was performed. When a significant result was obtained in the ANOVA test, a post-hoc analysis (Scheffé's test) was carried out to perform multiple comparisons.

In all cases, statistical significance was defined as $p<$ 0.05 .

\section{Results}

Patients and diagnoses

During the study period, 370 Caucasian patients (225 females) with headache were attended for the first time (new patients) in our headache clinic. The mean age was 43 years (range: 14-86 years). The final diagnoses of the patients are specified in Table 1. Of the participants, 172 (46.4\%) had migraine, 76 (20.5\%) tension-type headache, 13 patients $(3.5 \%)$ had cluster headache or other trigeminal autonomic cephalalgias and 22 (5.9\%) had other primary headaches. In our group, 70 patients (18.9\%) had secondary headaches and nine $(2.4 \%)$ other headache types (including two patients with primary trochlear headache). Forty patients (10, $8 \%$ ) were diagnosed as having $\mathrm{MOH}$. The headache most frequently associated with $\mathrm{MOH}$ was migraine in 27 patients $(67.5 \%$ of the cases of $\mathrm{MOH})$. In relation with headache frequency at admission, 125 patients $(33.7 \%)$ reported headache

Table 1 Diagnoses according to ICHD-2 and revised ICHD-2 criteria, given at the Headache Clinic of the University Clinic of Navarra

\begin{tabular}{|c|c|c|}
\hline Diagnoses & $\mathrm{n}$ & $\%$ \\
\hline Migraine & 172 & 46.4 \\
\hline Migraine without aura & 110 & 29.7 \\
\hline Migraine with aura & 42 & 11.3 \\
\hline Chronic migraine & 20 & 5.4 \\
\hline Tension-type headache $(\mathrm{TTH})$ & 76 & 20.8 \\
\hline Episodic infrequent TTH & 5 & 1.3 \\
\hline Episodic frequent TTH & 61 & 16.4 \\
\hline Chronic TTH & 10 & 2.7 \\
\hline $\begin{array}{l}\text { Cluster headache and other trigeminal autonomic } \\
\text { cephalalgias }\end{array}$ & 13 & 3.5 \\
\hline Cluster headache & 12 & 3.2 \\
\hline Paroxysmal hemicrania & 1 & 0.2 \\
\hline Other primary headaches & 22 & 5.9 \\
\hline Primary stabbing headache & 7 & 1.9 \\
\hline Primary cough headache & 1 & 0.2 \\
\hline Primary exertional headache & 2 & 0.5 \\
\hline Primary thunderclap headache & 2 & 0.5 \\
\hline Hemicrania continua & 2 & 0.5 \\
\hline New daily-persistent headache & 8 & 2.1 \\
\hline Secondary headaches & 70 & 18.9 \\
\hline Medication-overuse headache & 40 & 10.8 \\
\hline Cervicogenic headache & 15 & 4.0 \\
\hline Other secondary headaches & 15 & 4.0 \\
\hline Cranial neuralgias and central causes of facial pain & 8 & 2.1 \\
\hline Trigeminal neuralgia & 5 & 1.3 \\
\hline Occipital neuralgia & 3 & 0.8 \\
\hline Other headache types & 9 & 2.4 \\
\hline Total & 370 & 100 \\
\hline
\end{tabular}

International Classification of Headache Disorders, second edition (ICHD-2); n: number of patients; \%: percentages. 
more than 15 days per month in the previous three months.

\section{RM and R-CM}

19 patients (11 women) fulfilled the diagnostic criteria for RM or R-CM, representing $5.1 \%$ of all patients. The features of RM or R-CM are summarised in Table 2. Of the 19 patients, four (one woman) met the criteria for RM, while 15 patients (10 women) met the criteria for $\mathrm{R}-\mathrm{CM}$. The mean age of refractory migraineurs was 43 years (range, 26-66 years)

All refractory migraineurs had undergone abortive treatments with triptans and NSAID, except one patient who was allergic to NSAID. Intranasal or injectable dihydroergotamine was not used in our patients because this drug is not available in Spain. All possible triggers and lifestyle factors were addressed.

All refractory migraineurs had undergone a therapeutic trial with more than 2 groups of preventive drugs for at least 2 months at optimal or maximal tolerated doses, unless terminated early due to adverse effects. 17 patients had received tricyclic antidepressants (mainly amitriptyline, but also nortriptyline), 14 patients had received beta-blockers (mainly propranolol, but also atenolol and metoprolol), 16 patients had received calciumchannel-blockers (mainly flunarizine) and 18 patients had received anticonvulsants (14 topiramate, 10 valproate, 5 gabapentin, 2 lamotrigine, 1 pregabaline, 1 carbamazepine and 1 zonisamide). Three patients experienced intolerance to preventive drugs (topiramate in 3 and amitriptyline in 1).

Table 2 RM and R-CM characteristics*

\begin{tabular}{lc}
\hline Feature & $\begin{array}{c}\text { Study group } \\
(\mathbf{n}=19)\end{array}$ \\
\hline Sex & $11(57.9 \%)$ \\
$\quad$ Female & $8(42.1 \%)$ \\
$\quad$ Male & $43(26-66)$ \\
Age, mean (range) & $22.7(13-36)$ \\
Age at headache onset, mean (range) & \\
Type of migraine & \\
$\quad$ Episodic migraine & $4(21.1 \%)$ \\
Chronic migraine & $15(78.9 \%)$ \\
MIDAS score, mean (range) & $96(45-180)$ \\
Medication overuse & \\
$\quad$ Yes & \\
No & $7(36.8 \%)$ \\
\hline
\end{tabular}

*Values are number (percentage) unless otherwise stated
Additionally some patients had received other preventive treatments, with at least one positive randomized controlled trial in migraine, but not included in the current proposed criteria for RM and R-CM $[6,7]$. These additional treatments include botulinum toxin infiltration in 3 patients, pizotifen in 1, and vitamin B2 in 1. These treatments were not taken into account in establishing the diagnosis of RM or R-CM. Other common treatments used in refractory migraineurs to manage comorbid psychiatric disorders included serotonine reuptake inhibitors in 7 patients and lithium in 3 patients. Two patients had also received treatment with occipital nerve blocks.

The mean MIDAS score in refractory migraineurs was 96 (45-180), and therefore, in these cases the diagnosis of RM and R-CM may be qualified with the modifier "with significant disability".

In our headache clinic, $36,8 \%$ of patients with RM or $\mathrm{R}-\mathrm{CM}$ overused analgesics and therefore in these cases the diagnosis of RM and R-CM may be qualified with the modifier "with medication overuse".

For comparison purposes, refractory migraineurs were classified into four groups: RM without $\mathrm{MOH}, \mathrm{RM}$ with $\mathrm{MOH}, \mathrm{R}-\mathrm{CM}$ without $\mathrm{MOH}$ and R-CM with $\mathrm{MOH}$ (Table 3). No statistical differences between the groups were found when comparing for sex, age, age of headache onset, preventive drugs and abortive medicines. Only when comparing the MIDAS scores, differences between the three groups were statistically significant ( $F$ $=11.37, p=0.001$ ). Sheffé's test demonstrated that differences in MIDAS scores were significant $(p=0.001)$ when comparing the groups of episodic RM with $\mathrm{MOH}$ (mean MIDAS of 65) or RM without $\mathrm{MOH}$ (mean MIDAS of 56) against the group of R-CM with $\mathrm{MOH}$ (mean MIDAS of 131.6). The differences in MIDAS scores were also significant $(p=0.02)$ when comparing the group of R-CM with $\mathrm{MOH}$ (mean MIDAS of 131.6) against the group of $\mathrm{R}-\mathrm{CM}$ without $\mathrm{MOH}$ (mean MIDAS of 92.5).

\section{Discussion}

$\mathrm{RM}$ and R-CM accounted for $5.1 \%$ of patients attended for the first time in our headache unit. To our knowledge, this is the first study to describe the frequency of refractory migraineurs in a headache unit with the current diagnostic criteria. The proposed criteria for both $\mathrm{RM}$ and R-CM introduce the new term "refractory" into the classification of chronic headache [12-14]. The ICHD-2 diagnostic criteria do not provide any usable classification for this relatively common condition between the patients attended in tertiary headache units. Refractory headache patients are usually referred to specialized clinics [15] and most of them require follow-up visits for several months. Headache specialists intuitively 
Table 3 RM and R-CM subgroups characteristics

\begin{tabular}{|c|c|c|c|c|}
\hline & $\begin{array}{c}\text { RM } \\
\text { without } \mathrm{MOH}\end{array}$ & $\begin{array}{c}\text { RM } \\
\text { with } \mathrm{MOH}\end{array}$ & $\begin{array}{c}\text { R-CM } \\
\text { without } \mathrm{MOH}\end{array}$ & $\begin{array}{c}\text { R-CM } \\
\text { with } \mathrm{MOH}\end{array}$ \\
\hline $\mathrm{n}$ & 2 & 2 & 10 & 5 \\
\hline Age, mean (range) & $34.5(24-43)$ & $38(28-48)$ & $42.3(31-60)$ & $49.8(36-66)$ \\
\hline MIDAS score, mean (range) & $56(50-62)$ & $65(50-80)$ & $92.5(45-105)$ & $131.6(94-180)$ \\
\hline
\end{tabular}

$\mathrm{MOH}$ : Medication Overuse Headache; n: number of patients; RM: refractory migraine; R-CM: refractory chronic migraine

recognize when a patient is refractory, but with the proposed criteria for both RM and R-CM is possible to define the frequency of this patient population. The benefits of the newly proposed diagnostic criteria are not only the possibility to study the epidemiology of RM and R-CM, but also to define the treatment strategies for these challenging patients.

The overall age and sex distribution among headache patients in our headache unit is similar to previous studies in other headache units in Spain [16]. In our RM and R-CM patients, headaches caused a significant interference with function and quality of life. All our RM and $\mathrm{R}-\mathrm{CM}$ patients experienced severe disability (MIDAS $\geq$ 21) and therefore the diagnosis of RM and R-CM may be qualified with the modifier "with significant disability". The noteworthy MIDAS found in refractory migraineurs is consistent with the scores observed in those migraneurs treated with neurostimulation after the failure of several preventive drugs [17], and may be related to the significant degree of disability caused by refractory pain. Moreover, chronic migraine is more disabling than episodic migraine in the population [3], and likewise in our sample, the highest MIDAS corresponds to R-CM. Our study shows that the proportion of patients with chronic migraine is very similar to the proportion of RM and R-CM. These findings can be explained because refractory migraineurs may suffer from episodic or chronic migraine. Furthermore, in the chronic migraine revised ICHD-2 criteria [11], medication-overuse has to be ruled out, and therefore some migraneurs with near daily headache have to be classified as having $\mathrm{MOH}$. Between our patients classified as having medication-overuse headache, most were migraineurs. According to revised ICHD-2 criteria [17], if a patient has headache on $\geq 15$ days/month after $>3$ months of regular overuse of one or more acute/symptomatic treatment drugs, and the headache has developed or markedly worsened during medication overuse, then a diagnosis of $\mathrm{MOH}$ can be made. If the headache persists after 2 months of withdrawal, then a new diagnosis of chronic migraine is given. Considering that definition of RM and $\mathrm{R}-\mathrm{CM}$ was to have worldwide application, it was not considered practical to avoid medication overuse. For that reason, medication overuse was considered a modifier, although, ideally, medications potentially causing
MOH must be withdrawn before a patient with migraine can be identified as refractory.

Our study has some evident limitations. The best approach to estimate the proportion of patients with $\mathrm{RM}$ and $\mathrm{R}-\mathrm{CM}$ is a population based study and not a single center study, such as the present one. However, the information regarding the failure of adequate trials of acute treatments and preventive drugs (required for the diagnosis of RM and R-CM) is difficult to obtain by means of questionnaires or phone surveys, which are the usual methods in population-based studies. This sort of information is best obtained in person by an experienced neurologist with enough time to obtain the history, and review the clinical records, as occurs in most of headache units. Also, our data were obtained from a single headache unit and therefore our findings may not be representative of other tertiary headache centres. However, the proportion of patients with chronic headache and the distribution of diagnoses observed in the present study are similar to other headache units. In our sample, and also in several series of patients attended in headache units, patients with chronic headaches were over-represented [18]. Concerning headache diagnoses, the high frequency of tensiontype headache observed in our population, but also in previous reports $[16,19,20]$, contrasts with the experience of other headache units $[21,22]$. These differences may be partially explained by the pattern of referral to the headache unit (some of our patients are selfreferred) and the application of ICHD-II criteria in the present study. In fact, the distribution of diagnoses in the present work is similar to headache units in which patients were classified according to ICHD-2 [16,23]. Recall bias may constitute an additional limitation and may have affected our results. Patients might have had problems remembering the preventive therapeutic groups tried, or whether the trial was adequate, which is needed for the definition of RM and R-CM. However, preventive drugs were used during prolonged periods of time and most of the patients remembered details of the trials; in the remaining cases, such information was obtained from medical records.

The reliability and external validity of the proposed diagnostic criteria for RM need to be established in future studies. We consider that the present criteria are 
valid and exhaustive, but there are two shortcomings. First, the use of ergotics, even in oral presentation, in patients with migraine is not common [24]. In Spain and other countries, DHE is not available either intranasal or injectable forms. Thus, our patients do not meet the criterion that a trial with DHE (intranasal or injectable formulation) is needed for RM or R-CM diagnosis. Given that the definitions of RM and R-CM are going to be used worldwide, and the unavailability intranasal or injectable DHE in several countries, this criterion must be reviewed. In addition, $\mathrm{MOH}$ is included in the proposed criteria as a modifier and patients can be classified as having R-CM with or without $\mathrm{MOH}$. We agree that diagnoses of RM and R-CM may be made even in patients who overuse analgesics. However, we would specify in the criteria that overused medication needs to be withdrawn for two months on at least one occasion, before the patient can be classified as refractory.

\section{Conclusion}

In conclusion, $\mathrm{RM}$ and $\mathrm{R}-\mathrm{CM}$ are relatively common conditions among patients evaluated for the first time in a headache unit. Patients with RM and R-CM are severely disabled and need to be cared by neurologists attached to specialized headache clinics. The proposed operational criteria may be useful not only to identify those patients who require care in headache units but also to select the candidates for combinations of prophylactic drugs or invasive treatments, such as neurostimulation, and to facilitate clinical studies in this patient group.

\section{Funding}

None received.

\section{List Of Abbreviations}

(RM): Refractory migraine; (MIDAS): Migraine Disability Assessment Score; (ICHD-2): International Classification of Headache Disorders, second edition; (NSAID): Non-Steroidal Anti-Inflammatory Drugs; (R-CM): Refractory chronic migraine; $(\mathrm{MOH})$ : Medication overuse headache.

\section{Authors' contributions}

PI: involved in the conception and design, analysis and interpretation of data, drafting the manuscript and revision for important intellectual content and has read and approved the final manuscript. JAP: involved in acquisition of data, analysis and interpretation of data, drafting the manuscript, revision for important intellectual content and has read and approved the final manuscript. RFT: involved in acquisition of data, revision for important intellectual content and has read and approved the final manuscript. EMV: involved in the conception and design, and revision for important intellectual content and has read and approved the final manuscript.

\section{Competing interests}

The authors declare that they have no competing interests.

Received: 23 June 2011 Accepted: 1 August 2011

Published: 1 August 2011

\section{References}

1. Headache Classification Committee of the International Headache Society: The international classification of headache disorders. Cephalalgia , 2 2004, 24(Suppl 1):9-160.

2. Lipton RB, Stewart WF, Diamond S, Diamond ML, Reed M: Prevalence and burden of migraine in the united states: Data from the American migraine study ii. Headache 2001, 41:646-657.

3. Bigal ME, Serrano D, Reed M, Lipton RB: Chronic migraine in the population: Burden, diagnosis, and satisfaction with treatment. Neurology 2008, 71:559-566.

4. Goadsby PJ, Sprenger T: Current practice and future directions in the prevention and acute management of migraine. Lancet Neurol 2010, 9:285-298.

5. Goadsby PJ, Schoenen J, Ferrari MD, Silberstein SD, Dodick D: Towards a definition of intractable headache for use in clinical practice and trials. Cephalalgia 2006, 26:1168-1170.

6. Schulman EA, Lake AE, Goadsby PJ, Peterlin BL, Siegel SE, Markley HG, Lipton RB: Defining refractory migraine and refractory chronic migraine: Proposed criteria from the refractory headache special interest section of the american headache society. Headache 2008, 48:778-782.

7. Schulman EA, Peterlin BL, Lake AE, Lipton RB, Hanlon A, Siegel S, Levin M, Goadsby PJ, Markley HG: Defining refractory migraine: Results of the rhsis survey of American Headache Society members. Headache 2009, 49:509-518.

8. Rothrock JF: Treatment-refractory chronic migraine: The ogre emerges from the shadows. Headache 2009, 49:631-633.

9. Lipton RB, Bigal ME: Toward an epidemiology of refractory migraine: Current knowledge and issues for future research. Headache 2008, 48:791-798.

10. Lipton RB, Stewart WF, Sawyer J, Edmeads JG: Clinical utility of an instrument assessing migraine disability: The migraine disability assessment (MIDAS) questionnaire. Headache 2001, 41:854-861.

11. Olesen J, Bousser MG, Diener HC, Dodick D, First M, Goadsby PJ, Gobel H, Lainez MJ, Lance JW, Lipton RB, Nappi G, Sakai F, Schoenen J, Silberstein SD, Steiner TJ: New appendix criteria open for a broader concept of chronic migraine. Cephalalgia 2006, 26:742-746.

12. Bussone G: Clinical considerations on chronic migraine, pharmacoresistance and refractoriness. Neurol Sci 2010, 31(Suppl 1): S83-85.

13. D'Amico D, Leone M, Grazzi L, Bussone G: When should "chronic migraine" patients be considered "refractory" to pharmacological prophylaxis? Neurol Sci 2008, 29(Suppl 1):S55-58.

14. Schulman EA, Brahin EJ: Refractory headache: Historical perspective, need, and purposes for an operational definition. Headache 2008, 48:770-777.

15. Mathew NT: Chronic refractory headache. Neurology 1993, 43:S26-33.

16. Guerrero AL, Rojo E, Herrero S, Neri MJ, Bautista L, Penas ML, Cortijo E, Mulero P, Fernandez R: Characteristics of the first 1000 headaches in an outpatient headache clinic registry. Headache 2001, 51:226-231.

17. Popeney CA, Aló KM: Peripheral neurostimulation for the treatment of chronic, disabling transformed migraine. Headache 2003, 43:369-75.

18. Sanin LC, Mathew NT, Bellmeyer LR, Ali S: The international headache society (IHS) headache classification as applied to a headache clinic population. Cephalalgia 1994, 14:443-446.

19. Srikiatkhachorn A, Phanthumchinda K: Prevalence and clinical features of chronic daily headache in a headache clinic. Headache 1997, 37:277-280.

20. Gesztelyi G, Bereczki D: Primary headaches in an outpatient neurology headache clinic in East Hungary. Eur J Neurol 2004, 11:389-395. 
21. Silberstein SD, Silberstein JR: Chronic daily headache: Long-term prognosis following inpatient treatment with repetitive IV DHE. Headache 1992, 32:439-445.

22. Bigal ME, Sheftell FD, Rapoport AM, Lipton RB, Tepper SJ: Chronic daily headache in a tertiary care population: Correlation between the international headache society diagnostic criteria and proposed revisions of criteria for chronic daily headache. Cephalalgia 2002, 22:432-438.

23. Jensen $R$, Zeeberg $P$, Dehlendorff $C$, Olesen J: Predictors of outcome of the treatment programme in a multidisciplinary headache centre. Cephalalgia 2010, 30:1214-1224.

24. Bigal ME, Borucho S, Serrano D, Lipton RB: The acute treatment of episodic and chronic migraine in the USA. Cephalalgia 2009, 29:891-897.

Pre-publication history

The pre-publication history for this paper can be accessed here: http://www.biomedcentral.com/1471-2377/11/94/prepub

doi:10.1186/1471-2377-11-94

Cite this article as: Irimia et al:: Refractory migraine in a headache clinic population. BMC Neurology 2011 11:94.

\section{Submit your next manuscript to BioMed Central} and take full advantage of:

- Convenient online submission

- Thorough peer review

- No space constraints or color figure charges

- Immediate publication on acceptance

- Inclusion in PubMed, CAS, Scopus and Google Scholar

- Research which is freely available for redistribution

Submit your manuscript at www.biomedcentral.com/submit 